\title{
Extension of Biot theory to the problem of saturated microporous elastic media with isolated cracks or/and vugs
}

\author{
J. Lewandowska, Laboratoire de Mécanique et Génie Civil (LMGC), UM2, CNRS, CC048 \\ Place Eugene Bataillon, 34095 Montpellier Cedex 5, France \\ J.-L. Auriault, Lab. 3S, UJF, INPG, CNRS, Domaine universitaire, \\ BP 53, 38041 Grenoble cedex 9, France
}

\begin{abstract}
The purpose of this paper is to develop the macroscopic model of hydro-mechanical coupling for the case of a porous medium containing isolated cracks or/and vugs . In the development we apply the asymptotic expansions homogenization method. It is shown that the general structure of Biot's model is the same as in the case of homogeneous medium but the poro-elastic parameters are modified. Two numerical examples are presented. They concern the computations of Biot's parameters in isotropic and anisotropic cases. It can also be seen how the presence of near-zerovolume cracks influences Biot's parameters of the porous matrix. It can significantly affect the coupled hydro-mechanical behaviour of damaged porous medium.
\end{abstract}

\section{Introduction}

Microporous media with cracks or/and vugs represent a class of natural and/or damaged geomaterials (soils, rocks or cement-based materials). They may be considered as special cases of double porosity media, if the criterion of classification is a large contrast between the average size of pores in the microporous matrix and the average size of the cracks or vugs. However, this criterion is insufficient for the precise description and classification when we analyze the coupled hydro-mechanical behaviour of saturated cracked media. It turns out that additional morphological information about the connectivity of cracks is needed. This conclusion comes from the study of hydraulic behaviour of microporous media with connected and disconnected cracks. "Diconnected" means here that the crack or vug system do not percolate in the absence of microporosity.

The homogenization using asymptotic developments [1] showed that the macroscopic fluid filtration in a rigid porous medium is very different in case of closed fissures, from the one when they are connected. When the fissures (cracks) are disconnected, the velocity of filtration depends on the average size of pores in the porous matrix. On the contrary, in case of connected fissures the porous matrix plays a secondary role (negligible) and the flow velocity is governed by the network of fissures at the first order of approximation.

In addition, in case of unsaturated water flow through a rigid porous medium with connected fissures it was shown by using the homogenization technique [2] that memory effects are present during the transient phase of flow : i.e. the macroscopic equivalent model is non-local in time. This kind of behaviour is commonly associated with the existence of double porosity. The memory effect is absent, if the fissures are disconnected $[3,4]$.

The modelling is also complex when the porous medium through which filtrates an incompressible fluid is deformable. The coupled hydro-mechanical behaviour of deformable double porosity media was studied by using the homogenization technique (asymptotic development method) in a series of papers $[5,6,7]$. In that analysis it was assumed that the porous medium contains fractures that 
are connected. By double changing of scales three different macroscopic models were obtained, according to the ratio between pores, fractures and global scales. One of these models shows memory effects which are related to fluid filtration through the micro-porosity.

Historically, the term double porosity was firstly introduced by Barenblatt, Zheltov and Kochina [8], who studied the effect of fissures and matrix porosity in the rock-mass- seepage problem by using the phenomenological approach. They introduced the assumption that the mass flow between the two porosity systems is determined by the pressure jump in the two systems. The further significant contributions are Warren and Root [9], and Wilson and Aifantis [10]. Afterwords, the modern homogenization theory for materials with periodic structures [11, 12], offered the possibility for a detailed analysis of the upscaling process and the relationship between the microstructure, in particular phase connectivity, and the macroscopic behaviour. It is also important that we can identify the local fields and to precise the domain of validity of the modelling.

There exist also in the literature a large number of contributions regarding other homogenization techniques. They are essentially applied to determine the poro-mechanical or hydraulic properties, and the constitutive laws of materials containing heterogeneities like cracks or vugs. Two groups of such homogenization techniques can be distinguished:

i) The homogenization method based on the analysis of the behaviour of an assumed pattern representing an idealized microstructure.Among them are: the self-consistent approach, the Mori-Tanaka scheme, etc...[13].

ii) The numerical homogenization methods that use the volume averaging of the behaviour of a statistical ensemble of particles (particle-type models). Among these methods one can cite: the discrete element method (DEM), the lattice Boltzmann (LB) method, the molecular dynamics method (MDM), the contact dynamics method (CDM). A review of the state of the art is presented in [14] The aim of this paper is to develop a macroscopic model of hydro-mechanical coupling for the case of a porous medium containing non-connected cracks (or vugs). Such a model will be an extension of the classical Biot model $[15,16]$. The analysis will start at the mesoscopic scale (i.e. the scale of the period $\Omega$ ). It will be assumed that at that scale the medium was subjected to a damage, so that a network of non-connected cracks (vugs) appeared. The poro-elastic behaviour will be studied by using the asymptotic expansions homogenization method. It will be shown that the structure of the macroscopic equivalent model is similar to the Biot's model that is assumed at the mesoscopic scale, but the effective parameters are modified. In particular, the hydromechanical coupling variables $\boldsymbol{\alpha}$ (Biot's tensor) and $\beta$ (Biot's coefficient) are significantly affected by the presence of cracks (vugs). The paper is organized as follows. In Section 2 the formulation of the problem at the mesoscopic scale is presented. Sections 3 and 4 show the homogenization process. A numerical example is presented in Section 5. Finally, Section 6 contains the conclusions.

\section{Crack (vug) scale description}

We consider an elastic saturated microporous medium which contains saturated cracks or vugs (macropores). Crack (or vug) volumes are not connected: the fluid flows from a crack (or vug) to another one through the microporous medium. The saturating fluid is viscous Newtonian and incompressible. And we look for the equivalent macroscopic behaviour of this two-phase medium at a scale $L$ which is large compared to the scale $l$ of the cracks (or vugs): $l / L=\varepsilon \ll 1$, when the micropore size $l_{p}$ itself is small with respect to the crack (vug) size, with a ratio $l_{p} / l=\varepsilon$. Due to this separation of scales, periodic and non-periodic media show similar macroscopic equivalent behaviour [17]. Therefore, without loss of generality, the heterogeneous medium is assumed as periodic of period $\Omega$. The microporous part of $\Omega$ is noted $\Omega_{m}, \Omega_{p}$ is the micropore domain at the pore scale and for the simplicity of the presentation the period contains a single crack (vug) $\Omega_{c} . \Gamma$ is the boundary of the crack (vug) (Figure 1). We admit Biot's model for the deformable microporous medium [16] 


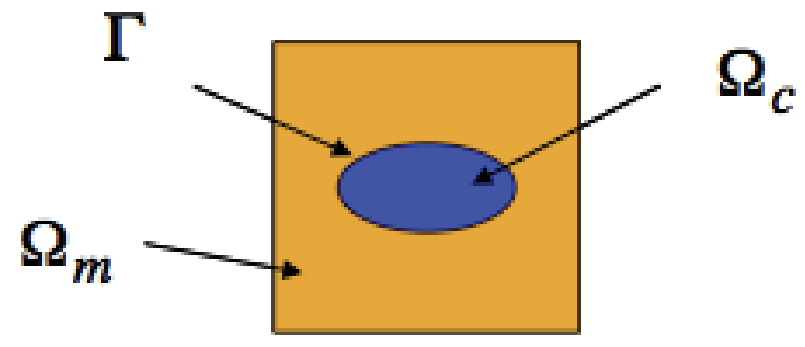

Figure 1: Schematic view of the period of a porous medium with a crack (vug)

$$
\frac{\partial \sigma_{i j}^{M}}{\partial X_{j}}=0
$$

where

$$
\begin{gathered}
\sigma_{i j}^{M}=\sigma_{i j}^{S}-\alpha p^{P}, \quad \sigma_{i j}^{S}=c_{i j k l} e_{X k l}\left(\mathbf{u}^{S}\right) \text { in } \Omega_{m}, \\
\frac{\partial}{\partial X_{i}}\left(v_{i}^{P}-\phi^{P} \frac{\partial u_{i}^{S}}{\partial t}\right)=-\alpha \frac{\partial e_{X i i}^{S}\left(\mathbf{u}^{S}\right)}{\partial t}-\beta \frac{\partial p^{P}}{\partial t}, \\
v_{i}^{P}-\phi^{P} \frac{\partial u_{i}^{S}}{\partial t}=-\frac{K}{\mu} \frac{\partial p^{P}}{\partial X_{i}} \text { in } \Omega_{c} .
\end{gathered}
$$

For the sake of simplicity, tensors $\boldsymbol{\alpha}$ and $\mathbf{K}$ are assumed as isotropic and $\alpha$ and $\beta$ are constant. $\mathbf{X}$ is the space variable, $\boldsymbol{\sigma}^{M}$ the total stress in $\Omega_{m}, \boldsymbol{\sigma}^{S}$ the partial solid stress in $\Omega_{m}, p^{P}$ the micropore pressure, $\mathbf{v}^{P}$ the average fluid velocity in $\Omega_{m}$ (We note $\mathbf{v}^{D}=\mathbf{v}^{P}-\phi^{P} \frac{\partial \mathbf{u}^{S}}{\partial t}$ is Darcy's velocity), $\mathbf{u}^{S}$ the displacement in the microporous matrix, $\mathbf{e}_{X}^{S}\left(\mathbf{u}^{S}\right)$ the corresponding deformation tensor, $\mathbf{c}$ the elastic tensor and $\phi^{P}=\Omega_{p} / \Omega_{m}$ the micro-porosity. Recall that the permeability is of the order $\mathbf{K}=\mathcal{O}\left(l_{p}^{2}\right)$, where $l_{p}$ is the micropore characteristic size. In the $\Omega_{c}$ domain, the fluid verifies the Stokes equation

$$
\begin{aligned}
\frac{\partial \sigma_{i j}^{C}}{\partial X_{j}}=0, \quad \sigma_{i j}^{C} & =2 \mu e_{X i j}\left(\mathbf{v}^{C}\right)-p^{C} I_{i j}, \\
\frac{\partial v_{i}^{C}}{\partial X_{i}} & =0 \text { in } \Omega_{c},
\end{aligned}
$$

where $\mathbf{v}^{C}$ is the fluid velocity in $\Omega_{c}, p^{C}$ the crack (vug) pressure, $\mathbf{e}_{X}\left(\mathbf{v}^{C}\right)$ is the rate of deformation tensor and $\mu$ is the dynamic viscosity,

On the boundary $\Gamma$ of the crack (vug), we have the continuity of the total stress and of the pressure

$$
\begin{gathered}
\sigma_{i j}^{M} n_{j}=\sigma_{i j}^{C} n_{j}, \\
p^{P}=p^{C},
\end{gathered}
$$

and the average velocities in $\Omega_{m}$ and $\Omega_{c}$ are equal (Auriault and Boutin, 1992)

$$
\underbrace{\left(\mathbf{v}^{P}-\phi^{P} \frac{\partial \mathbf{u}^{S}}{\partial t}\right)}_{\text {Guid velocity relative to the solid }}+\underbrace{\frac{\partial \mathbf{u}^{S}}{\partial t}}_{\text {Solid velocity }}=\mathbf{v}^{C} \text { on } \Gamma,
$$

where $\mathbf{n}$ is the unit normal to $\Gamma$ exterior to $\Omega_{m}$. 


\section{Estimations and dimensionless equations}

We consider a macroscopic sample of characteristic size $L$ made of a large number of periods $\Omega$, with a view to verfy $\epsilon=l / L \ll 1$. We assume that $\boldsymbol{\sigma}^{S}, p^{P}$ and $p^{C}$ are of similar order of magnitude. Displacements of the solid $S$ and the fluid are also considered of similar order of magnitude, $\partial \mathbf{u}^{S} / \partial t=\mathcal{O}\left(\mathbf{v}^{P}\right)=\mathcal{O}\left(\mathbf{v}^{C}\right)$. From (4) we have

$$
v^{P}=\mathcal{O}\left(\frac{l_{p}^{2}}{\mu} \frac{p^{P}}{L}\right)
$$

Therefore, the ratio of the viscous term to the pressure term in (5) can be evaluated at the macroscopic level as

$$
\left(\frac{\left|2 \mu e_{X i j}\left(\mathbf{v}^{C}\right)\right|}{\left|p^{C}\right|}\right)_{L}=\mathcal{O}\left(\frac{2 \mu v^{C}}{L p^{C}}\right)=\mathcal{O}\left(\frac{l_{p}^{2}}{L^{2}}\right)=\mathcal{O}\left(\varepsilon^{4}\right) .
$$

Finally, using $\mathbf{x}=\mathbf{X} / L$ as the dimensionless space variable and keeping similar notations for dimensionless physical quantities yield the dimensionless local description in the following form. We have in $\Omega_{m}$,

$$
\begin{gathered}
\frac{\partial \sigma_{i j}^{M}}{\partial x_{j}}=0 \\
\sigma_{i j}^{M}=\sigma_{i j}^{S}-\alpha p^{P}, \quad \sigma_{i j}^{S}=c_{i j k l} e_{x k l}\left(\mathbf{u}^{S}\right), \\
\frac{\partial}{\partial x_{i}}\left(v_{i}^{P}-\phi^{P} \frac{\partial u_{i}^{S}}{\partial t}\right)=-\alpha \frac{\partial e_{x i i}^{S}\left(\mathbf{u}^{S}\right)}{\partial t}-\beta \frac{\partial p^{P}}{\partial t}, \\
v_{i}^{P}-\phi^{P} \frac{\partial u_{i}^{S}}{\partial t}=-\frac{K}{\mu} \frac{\partial p^{P}}{\partial x_{i}},
\end{gathered}
$$

in $\Omega_{c}$,

$$
\begin{gathered}
\frac{\partial \sigma_{i j}^{C}}{\partial x_{j}}=0, \quad \sigma_{i j}^{C}=2 \varepsilon^{4} \mu e_{x i j}\left(\mathbf{v}^{C}\right)-p^{C} I_{i j}, \\
\frac{\partial v_{i}^{C}}{\partial x_{i}}=0
\end{gathered}
$$

and on $\Gamma$

$$
\begin{gathered}
\sigma_{i j}^{M} n_{j}=\sigma_{i j}^{C} n_{j}, \\
p^{P}=p^{C}, \\
\mathbf{v}^{C}=\mathbf{v}^{P}+\left(1-\phi^{P}\right) \frac{\partial \mathbf{u}^{S}}{\partial t} \text { on } \Gamma .
\end{gathered}
$$

\section{4 upscaling process}

By following the classical method of multiscale asymptotic expansions, we look for $\mathbf{u}^{S}, \mathbf{v}^{P}, p^{P}, \mathbf{v}^{C}$ and $p^{C}$ in the form

$$
\varphi=\varphi^{(0)}(\mathbf{x}, \mathbf{y}, t)+\varepsilon \varphi^{(1)}(\mathbf{x}, \mathbf{y}, t)+\varepsilon^{2} \varphi^{(2)}(\mathbf{x}, \mathbf{y}, t)+\cdots,
$$

where $\mathbf{y}=\mathbf{x} / \varepsilon$, and the $\varphi^{(i)}$ 's are $\Omega$-periodic with respect to $\mathbf{y}$. Variable $\mathbf{y}$ is well suited to describing the behaviour at the period scale whereas $\mathbf{x}$ shows the macroscopic variations at scale $L$. Introduction of such expansions in the system (10-18) and equating like powers of $\varepsilon$ yield boundary value problems to be investigated over the period $\Omega$. 


\subsection{Pressures $p^{P(0)}$ and $p^{C(0)}$}

Equations in (14) at the $\varepsilon^{-1}$ order yields

$$
\frac{\partial p^{C(0)}}{\partial y_{i}}=0, \quad \text { thus } \quad p^{C(0)}=p^{C(0)}(\mathbf{x}, t) .
$$

Equation (12) at the $\varepsilon^{-2}$ order and (17) at the $\varepsilon^{0}$ order give

$$
\frac{\partial}{\partial y_{i}}\left(\frac{K}{\mu} \frac{\partial p^{P(0)}}{\partial y_{i}}\right)=0 \text { in } \Omega_{m}, \quad p^{P(0)}=p^{C(0)} \text { on } \Gamma
$$

We deduce that

$$
p^{P(0)}=p^{C(0)}=p^{(0)}(\mathbf{x}, t) .
$$

\subsection{Displacements $\mathbf{u}^{S(0)}$ and $\mathbf{u}^{S(1)}$}

Equation (10) at the $\varepsilon^{-2}$ order and (16) at the $\varepsilon^{-1}$ order are in the form

$$
\frac{\partial}{\partial y_{i}}\left(c_{i j k l} e_{y k l}\left(\mathbf{u}^{S(0)}\right)\right)=0 \text { in } \Omega_{m}, \quad c_{i j k l} e_{y k l}\left(\mathbf{u}^{S(0)}\right) n_{j}=0 \text { on } \Gamma \text {. }
$$

Therefore

$$
\mathbf{u}^{S(0)}=\mathbf{u}^{S(0)}(\mathbf{x}, t) .
$$

Equation (10) at the $\varepsilon^{-1}$ order and (16) at the $\varepsilon^{0}$ order are written

$$
\begin{gathered}
\frac{\partial}{\partial y_{i}}\left(c_{i j k l}\left(e_{x k l}\left(\mathbf{u}^{S(0)}\right)+e_{y k l}\left(\mathbf{u}^{S(1)}\right)\right)\right)=0 \text { in } \Omega_{m}, \\
c_{i j k l}\left(e_{x k l}\left(\mathbf{u}^{S(0)}\right)+e_{y k l}\left(\mathbf{u}^{S(1)}\right)\right) n_{j}=-(1-\alpha) p^{(0)} n_{i} \text { on } \Gamma .
\end{gathered}
$$

That gives $\mathbf{u}^{S(1)}$ in the form

$$
u_{i}^{S(1)}=\xi_{i}^{l m}(\mathbf{y}) e_{x l m}\left(\mathbf{u}^{S(0)}\right)-\eta_{i}(\mathbf{y})(1-\alpha) p^{(0)}+\bar{u}^{S(1)}(\mathbf{x}, t),
$$

where $\bar{u}^{S(1)}(\mathbf{x}, t)$ is an $\mathbf{y}$-independent arbitrary displacement. The $\Omega$-periodic vector $\boldsymbol{\xi}^{p q}$, of zero volume average over $\Omega_{m}$, is the solution of the boundary value problem

$$
\begin{gathered}
\frac{\partial}{\partial y_{i}}\left(c_{i j k l}\left(\delta_{k p} \delta_{l q}+e_{y k l}\left(\boldsymbol{\xi}^{p q}\right)\right)\right)=0 \text { in } \Omega_{m}, \\
c_{i j k l}\left(\delta_{k p} \delta_{l q}+e_{y k l}\left(\boldsymbol{\xi}^{p q}\right)\right) n_{j}=0 \text { on } \Gamma,
\end{gathered}
$$

where $\delta_{k p}$ and $\delta_{l q}$ are Kronecker symbols.

And the $\Omega$-periodic vector $\boldsymbol{\eta}$, of zero volume average over $\Omega_{m}$, is the solution of

$$
\begin{gathered}
\frac{\partial}{\partial y_{i}}\left(c_{i j k l} e_{y k l}(\boldsymbol{\eta})\right)=0 \text { in } \Omega_{m}, \\
c_{i j k l} e_{y k l}(\boldsymbol{\eta}) n_{j}=n_{i} \text { on } \Gamma .
\end{gathered}
$$

Note that tensor $\boldsymbol{\xi}$ and vector $\boldsymbol{\eta}$ are similar to the ones obtained for saturated porous media showing one pore characteristic size, only, by considering domain $\Omega_{m}$ as non-porous (the classical Biot's model). Therefore they verify, see [17], p. 45, for details

$$
\int_{\Omega_{m}} \frac{\partial \xi_{i}^{l m}}{\partial y_{i}} \mathrm{~d} \Omega=-\int_{\Omega_{m}} c_{i j l m} e_{y i j}(\boldsymbol{\eta}) \mathrm{d} \Omega .
$$




\subsection{First pressure correctors $p^{P(1)}$ and $p^{C(1)}$}

The pressure $p^{C(1)}$ is obtained from (14) at the order $\varepsilon^{0}$

$$
\frac{\partial p^{(0)}}{\partial x_{i}}+\frac{\partial p^{C(1)}}{\partial y_{i}}=0, \quad \text { thus } \quad p^{C(1)}=-y_{i} \frac{\partial p^{(0)}}{\partial x_{i}}+\bar{p}^{C(1)}(\mathbf{x}, t)
$$

where $\bar{p}^{C(1)}(\mathbf{x}, t)$ is a $\mathbf{y}$-independent arbitrary function. The pressure $p^{P(1)}$ is the solution of (12) at the order $\varepsilon^{-1}$ with (17) at the $\varepsilon$ order

$$
\begin{gathered}
\frac{\partial}{\partial y_{i}}\left(\frac{K}{\mu}\left(\frac{\partial p^{(0)}}{\partial x_{i}}+\frac{\partial p^{P(1)}}{\partial y_{i}}\right)\right)=0 \text { in } \Omega_{m}, \\
p^{P(1)}=p^{C(1)}=-y_{i} \frac{\partial p^{(0)}}{\partial x_{i}}+\bar{p}^{C(1)}(\mathbf{x}, t) \text { on } \Gamma .
\end{gathered}
$$

That gives

$$
p^{P(1)}=-\tau_{i}(\mathbf{y}) \frac{\partial p^{(0)}}{\partial x_{i}}+\bar{p}^{P(1)}(\mathbf{x}, t)
$$

where $\bar{p}^{P(1)}(\mathbf{x}, t)$ is a $\mathbf{y}$-independent arbitrary function, and

$$
\bar{p}^{C(1)}(\mathbf{x}, t)=\bar{p}^{P(1)}(\mathbf{x}, t)=\bar{p}^{(1)}(\mathbf{x}, t) .
$$

The $\Omega$-periodic component $\tau_{p}$, of zero volume average over $\Omega_{m}$, is the solution of the boundary value problem

$$
\begin{gathered}
\frac{\partial}{\partial y_{i}}\left(\frac{K}{\mu}\left(\delta_{i p}+\frac{\partial \tau_{p}}{\partial y_{i}}\right)\right)=0 \text { in } \Omega_{m} \\
\tau_{p}=y_{p} \text { on } \Gamma .
\end{gathered}
$$

\subsection{Macroscopic momentum balance}

Consider (10) and (14) at the $\varepsilon^{0}$ order

$$
\begin{gathered}
\frac{\partial}{\partial y_{i}}\left(c_{i j k l}\left(e_{x k l}\left(\mathbf{u}^{S(1)}\right)+e_{y k l}\left(\mathbf{u}^{S(2)}\right)-\alpha I_{i j} p^{P(1)}\right)\right. \\
+\frac{\partial}{\partial x_{i}}\left(c_{i j k l}\left(e_{x k l}\left(\mathbf{u}^{S(0)}\right)+e_{y k l}\left(\mathbf{u}^{S(1)}\right)-\alpha I_{i j} p^{(0)}\right)=0 \text { in } \Omega_{m}\right. \\
\frac{\partial p^{(0)}}{\partial x_{i}}+\frac{\partial p^{C(1)}}{\partial y_{i}}=0 \text { in } \Omega_{c} .
\end{gathered}
$$

Let us integrate (37) on $\Omega_{m}$ and (38) on $\Omega_{c}$, respectively, and apply the divergence theorem. We obtain

$$
\begin{gathered}
\left\langle\frac{\partial}{\partial x_{i}}\left(c_{i j k l}\left(e_{x k l}\left(\mathbf{u}^{S(0)}\right)+e_{y k l}\left(\mathbf{u}^{S(1)}\right)-\alpha I_{i j} p^{(0)}\right)\right\rangle=\right. \\
-\frac{1}{|\Omega|} \int_{\Gamma}\left(c_{i j k l}\left(e_{x k l}\left(\mathbf{u}^{S(1)}\right)+e_{y k l}\left(\mathbf{u}^{S(2)}\right)\right)-\alpha p^{P(1)}\right) n_{j} \mathrm{~d} \Gamma \\
\left\langle\frac{\partial p^{(0)}}{\partial x_{i}}\right\rangle=\frac{1}{|\Omega|} \int_{\Gamma} p^{C(1)} n_{i} \mathrm{~d} \Gamma .
\end{gathered}
$$

$|\Omega|$ denotes the volume of $\Omega$ and the symbol $\langle a\rangle$ stands for the volume average of $a$

$$
\langle a\rangle=\frac{1}{|\Omega|} \int_{\Omega} a \mathrm{~d} \Omega .
$$


Now, subtracting member to member the second equation from the first one and using (16) at the $\varepsilon$ order

$$
\left(c_{i j k l}\left(e_{x k l}\left(\mathbf{u}^{S(1)}\right)+e_{y k l}\left(\mathbf{u}^{S(2)}\right)\right)-\alpha p^{P(1)}\right) n_{j}=-p^{C(1)} n_{i} \text { on } \Gamma,
$$

give the macroscopic momentum balance in the form

$$
\frac{\partial \sigma_{i j}^{T(0)}}{\partial x_{j}}=0
$$

where the $\boldsymbol{\sigma}_{i j}^{T(0)}=c_{i j k l}^{e f f} e_{x k l}\left(\mathbf{u}^{S(0)}\right)-\alpha_{i j}^{e f f} p^{(0)}$ are the components of the macroscopic total stress in the microporous medium with cracks. The effective tensors $\boldsymbol{\xi}^{e f f}$ and $\boldsymbol{\alpha}^{e f f}$ are defined by

$$
\begin{gathered}
c_{i j k l}^{e f f}=\left\langle c_{i j k l}+c_{i j m n} e_{y m n}\left(\boldsymbol{\xi}^{k l}\right)\right\rangle, \\
\alpha_{i j}^{e f f}=\left(\phi^{C}+\left(1-\phi^{C}\right) \alpha\right) I_{i j}+\left\langle(1-\alpha) c_{i j k l} e_{y k l}(\boldsymbol{\eta})\right\rangle .
\end{gathered}
$$

In the above equations $\phi^{C}=\Omega_{c} / \Omega$ is the volumetric fraction of the cracks (vugs).

Remark

Consider penny-shaped cracks $\mathcal{O}(l)$, of small thickness such that $\phi_{c}$ is small. Then we have

$$
\alpha_{i j}^{e f f} \approx \alpha I_{i j}+\left\langle(1-\alpha) c_{i j k l} e_{y k l}(\boldsymbol{\eta})\right\rangle \neq \alpha I_{i j},
$$

which means that the presence of cracks $\mathcal{O}(l)$, but with negligible volume influences the poromechanical behaviour of the medium.

\subsection{Macroscopic flow law}

Equation (13) at the order $\varepsilon^{0}$ gives

$$
v_{i}^{P(0)}-\phi^{P} \frac{\partial u_{i}^{S(0)}}{\partial t}=-\frac{K}{\mu}\left(\frac{\partial p^{P(1)}}{\partial y_{i}}+\frac{\partial p^{(0)}}{\partial x_{i}}\right) .
$$

Consider a parallelepipedic period with surfaces perpendicular to the axes. It is easy to check that the surface average fluid flow $\left\langle v_{i}^{P(0)}\right\rangle_{S}$ through any of the period surface $S_{i}$ normal to $y_{i}$ can be written at the first order of approximation in the form

$$
\left\langle v_{i}^{P(0)}\right\rangle_{S}=\frac{1}{|\Omega|} \int_{\partial \Omega} y_{i} v_{k}^{P(0)} n_{k} \mathrm{~d} S .
$$

Thus, multiplying (43) with $i=k$ by $y_{i}$ and integrating over the period surface yield with the help of the definition $(36)$ of $p^{P(1)}$ to the macroscopic flow law

$$
v_{i}^{D(0)}=\left\langle v_{i}^{P(0)}\right\rangle_{S}-\phi^{P} \frac{\partial u_{i}^{S(0)}}{\partial t}=-\frac{K_{i j}^{e f f}}{\mu} \frac{\partial p^{(0)}}{\partial x_{j}},
$$

where $\mathbf{v}^{D(0)}$ is the first order Darcy's velocity and the effective permeability $\mathbf{K}^{\text {eff }}$ is defined, after using (36), as

$$
K_{i j}^{e f f}=\frac{1}{|\Omega|} \int_{\partial \Omega} \frac{K}{\mu} y_{i}\left(I_{j l}-\frac{\partial \tau_{j}}{y_{l}}\right) n_{l} \mathrm{~d} S .
$$

This result was already obtained by Levy [1], in the case of a rigid microporous matrix with cracks. The macroscopic permeability depends on the matrix permeability and on the microstructure (through the solution for the vedtor $\tau$ ). 


\subsection{Macroscopic mass balance}

Balances (12) and (15) at the order $\varepsilon^{0}$ write

$$
\begin{gathered}
\frac{\partial}{\partial x_{i}}\left(v_{i}^{P(0)}-\phi^{P} \frac{\partial u_{i}^{S(0)}}{\partial t}\right)+\frac{\partial}{\partial y_{i}}\left(v_{i}^{P(1)}-\phi^{P} \frac{\partial u_{i}^{S(1)}}{\partial t}\right) \\
=-\alpha\left(\frac{\partial e_{x i i}^{S}\left(\mathbf{u}^{S(0)}\right)}{\partial t}+\frac{\partial e_{y i i}^{S}\left(\mathbf{u}^{S(1)}\right)}{\partial t}\right)-\beta \frac{\partial p^{(0)}}{\partial t} \text { in } \Omega_{m}, \\
\frac{\partial v_{i}^{C(0)}}{\partial x_{i}}+\frac{\partial v_{i}^{C(1)}}{\partial y_{i}}=0 \text { in } \Omega_{c},
\end{gathered}
$$

Let us average the two above equations on their respective domain of definition and let us add member to member the resulting relations. We obtain

$$
\frac{\partial A_{i}}{\partial x_{i}}+B=\left(1-\phi^{C}\right)\left(\phi^{P}-\alpha\right) \frac{\partial e_{x i i}\left(\mathbf{u}^{S(0)}\right)}{\partial t}-\beta\left(1-\phi^{C}\right) \frac{\partial p^{(0)}}{\partial t},
$$

with

$$
\begin{gathered}
A_{i}=\frac{1}{|\Omega|}\left(\int_{\Omega_{m}} v_{i}^{P(0)} \mathrm{d} \Omega+\int_{\Omega_{c}} v_{i}^{C(0)} \mathrm{d} \Omega\right), \\
B=\frac{1}{|\Omega|}\left(\int_{\Omega_{m}} \frac{\partial}{\partial y_{i}}\left(v_{i}^{P(1)}-\phi^{P} \frac{\partial u_{i}^{S(1)}}{\partial t}\right) \mathrm{d} \Omega+\int_{\Omega_{c}} \frac{\partial v_{i}^{C(1)}}{\partial y_{i}} \mathrm{~d} \Omega\right) .
\end{gathered}
$$

Equations (12) and (15) at the $\varepsilon^{-1}$ order give

$$
\frac{\partial v_{i}^{P(0)}}{\partial y_{i}}=0, \quad \frac{\partial v_{i}^{C(0)}}{\partial y_{i}}=0
$$

Therefore

$$
\begin{gathered}
|\Omega| A_{i}=\int_{\Omega_{m}} \frac{\partial}{\partial y_{j}}\left(y_{i} v_{j}^{P(0)}\right) \mathrm{d} \Omega+\int_{\Omega_{c}} \frac{\partial}{\partial y_{j}}\left(y_{i} v_{j}^{C(0)}\right) \mathrm{d} \Omega= \\
\int_{\Gamma} y_{i}\left(v_{j}^{P(0)}-v_{j}^{C(0)}\right) n_{i} \mathrm{~d} \mathrm{~S}+\int_{\partial \Omega} y_{i} v_{j}^{P(0)} n_{j} \mathrm{dS} .
\end{gathered}
$$

We have

$$
\int_{\partial \Omega} y_{i} v_{j}^{P(0)} n_{j} \mathrm{dS}=|\Omega|\left\langle v_{i}^{P(0)}\right\rangle_{S}
$$

and from (18)

$$
v_{j}^{P(0)}-v_{j}^{C(0)}=\left(\phi_{p}-1\right) \frac{\partial u_{j}^{S(0)}}{\partial t} \text { on } \Gamma \text {. }
$$

Therefore

$$
\begin{gathered}
|\Omega| A_{i}=|\Omega|\left\langle v_{i}^{P(0)}\right\rangle_{S}+\int_{\Gamma} y_{i}\left(\phi^{P}-1\right) \frac{\partial u_{j}^{S(0)}}{\partial t} n_{i} \mathrm{~d} S \\
=|\Omega|\left\langle v_{i}^{P(0)}\right\rangle_{S}+\left(1-\phi^{P}\right) \int_{\Omega_{c}} \frac{\partial}{\partial y_{j}}\left(y_{i} \frac{\partial u_{j}^{S(0)}}{\partial t}\right) \mathrm{d} \Omega . \\
A_{i}=\left\langle v_{i}^{P(0)}\right\rangle+\left\langle v_{i}^{C(0)}\right\rangle=\left\langle v_{i}^{P(0)}\right\rangle_{S}+\left(1-\phi^{P}\right) \phi^{C} \frac{\partial u_{i}^{S(0)}}{\partial t} .
\end{gathered}
$$

On an other hand

$$
|\Omega| B=\int_{\Omega_{m}} \frac{\partial}{\partial y_{i}}\left(v_{i}^{P(1)}-\phi^{P} \frac{\partial u_{i}^{S(1)}}{\partial t}\right) \mathrm{d} \Omega+\int_{\Omega_{c}} \frac{\partial v_{i}^{C(1)}}{\partial y_{i}} \mathrm{~d} \Omega=
$$




$$
\int_{\Gamma}\left(v_{i}^{P(1)}-\phi^{P} \frac{\partial u_{i}^{S(1)}}{\partial t}-v_{i}^{C(1)}\right) n_{i} \mathrm{dS}
$$

After using (18) at the $\varepsilon$ order

$$
v_{i}^{P(1)}-v_{i}^{C(1)}=\left(\phi^{P}-1\right) \frac{\partial u_{i}^{S(1)}}{\partial t} \text { on } \Gamma,
$$

it comes

$$
\begin{gathered}
|\Omega| B=-\int_{\Gamma} \frac{\partial u_{i}^{S(1)}}{\partial t} n_{i} \mathrm{~d} S=-\int_{\Omega_{m}} \frac{\partial e_{y i i}\left(\mathbf{u}^{S(1)}\right)}{\partial t} \mathrm{~d} \Omega . \\
B=-\left\langle e_{y i i}\left(\boldsymbol{\xi}^{l m}\right)\right\rangle \frac{\partial e_{x l m}\left(\mathbf{u}^{S(0)}\right)}{\partial t}+\left\langle e_{y i i}(\boldsymbol{\eta})\right\rangle(1-\alpha) \frac{\partial p^{(0)}}{\partial t}
\end{gathered}
$$

Introducing expressions (50) and (51) into (49) and using (45) yield the first order macroscopic mass balance in the form

$$
\frac{\partial}{\partial x_{i}}\left(v_{i}^{D(0)}\right)=-\gamma_{i j}^{e f f} \frac{\partial e_{x i j}\left(\mathbf{u}^{S(0)}\right)}{\partial t}-\beta^{e f f} \frac{\partial p^{(0)}}{\partial t},
$$

with

$$
\begin{gathered}
\gamma_{i j}^{e f f}=\left(\phi^{C}+\left(1-\phi^{C}\right) \alpha\right) I_{i j}-(1-\alpha)\left\langle e_{y l l}\left(\boldsymbol{\xi}^{i j}\right)\right\rangle, \\
\beta^{e f f}=\left(1-\phi^{C}\right) \beta+(1-\alpha)^{2}\left\langle e_{y i i}(\boldsymbol{\eta})\right\rangle .
\end{gathered}
$$

With the help of (32) we have

$$
\gamma_{i j}^{e f f}=\alpha_{i j}^{e f f} .
$$

The first order macroscopic model shows the classical Biot symmetry [16]. However, note that the values of the Biot parameters are strongly affected by the presence of cracks (vugs), see Section 5 Note that in the case of near-zero-volume cracks (vugs), when $\phi_{c} \approx 0$, then $\boldsymbol{\alpha}^{\text {eff }} \neq \alpha I_{i j}$ and $\beta^{e f f} \neq \beta$. It is an important result that confirms the influence of damage on the hydro-mechanical coupling.

\subsection{Dimensional equivalent macroscopic model}

Returning to dimensional quantities, the equivalent macroscopic model writes

$$
\begin{gathered}
\frac{\partial \sigma_{i j}^{T}}{\partial X_{j}}=\frac{\partial}{\partial X_{i}}\left(c_{i j k l}^{e f f} e_{X k l}\left(\mathbf{u}^{S}\right)-\alpha_{i j}^{e f f} p\right)=\mathcal{O}(\varepsilon), \\
\frac{\partial}{\partial X_{i}}\left(v_{i}^{D}\right)=-\alpha_{i j}^{e f f} \frac{\partial e_{X l l}\left(\mathbf{u}^{S}\right)}{\partial t}-\beta^{e f f} \frac{\partial p}{\partial t}+\mathcal{O}(\varepsilon), \\
v_{i}^{D}=-\frac{K_{i j}^{e f f}}{\mu} \frac{\partial p}{\partial X_{j}}+\mathcal{O}(\varepsilon) .
\end{gathered}
$$

The approximation $\mathcal{O}(\varepsilon)$ calls our attention to a generally imperfect separation of scales. As noted above in (55), the macroscopic model of the microporous medium with cracks (vugs) shows the symmetry of Biot's model which describes deformable single porosity media.

On an other hand we have from (50) and (45)

$$
\langle\mathbf{v}\rangle=\left\langle\mathbf{v}^{P}\right\rangle+\left\langle\mathbf{v}^{C}\right\rangle=\mathbf{v}^{D}+\left(\phi^{P}+\left(1-\phi^{P}\right) \phi^{C}\right) \frac{\partial \mathbf{u}^{S}}{\partial t}+\mathcal{O}(\varepsilon) .
$$

After noting that the porosity of the microporous media with cracks is

$$
\phi=\phi^{C}+\left(1-\phi^{C}\right) \phi^{P}=\phi^{P}+\left(1-\phi^{P}\right) \phi^{C},
$$




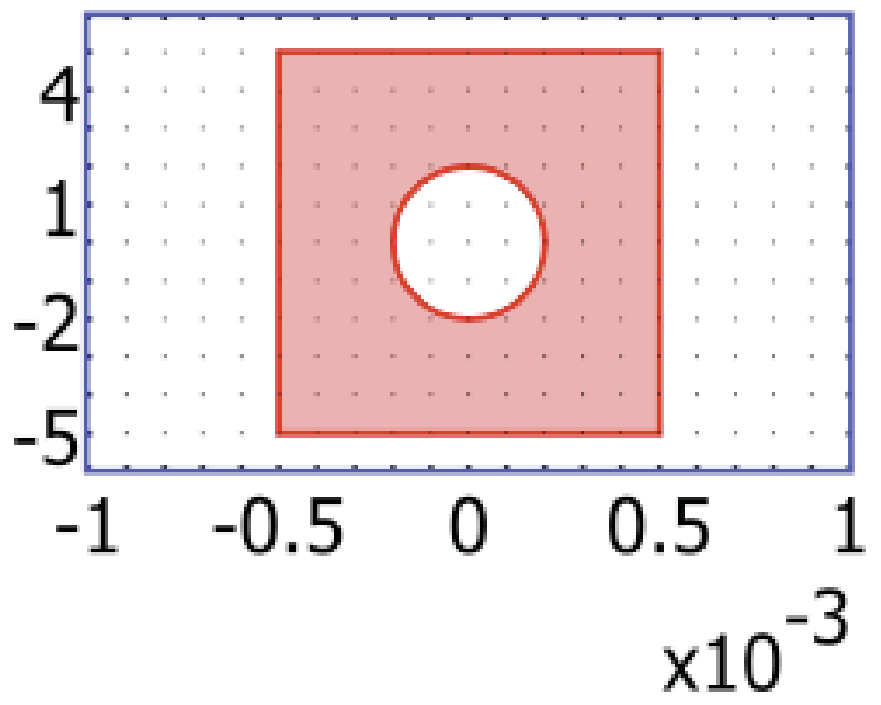

Figure 2: The microstructure of the porous medium with a circular vug.

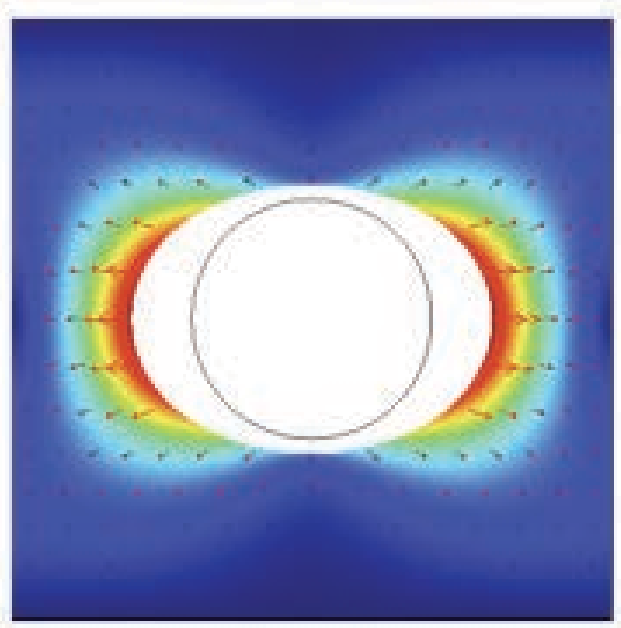

Figure 3: The solution $\boldsymbol{\xi}^{11}$ of the local problem (28-29) corresponding to a unit macroscopic strain in the $\mathrm{x}$-direction (horizontal). 


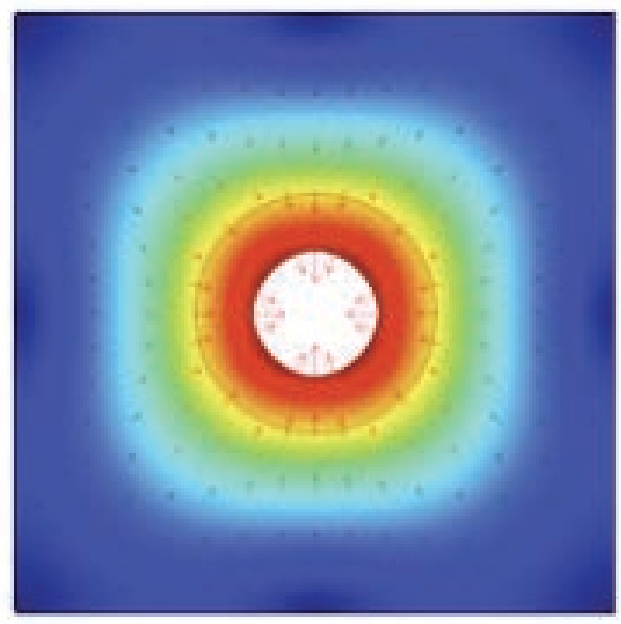

Figure 4: The solution $\boldsymbol{\eta}$ of the local problem (30-31) corresponding to $(1-\alpha) p^{(0)}=-1$ at the boundary $\Gamma$.

it comes

$$
\mathbf{v}^{D}=\langle\mathbf{v}\rangle-\phi \frac{\partial \mathbf{u}^{S}}{\partial t}+\mathcal{O}(\varepsilon)
$$

It is important to note that Darcy's flux $\mathbf{v}^{D}$ is related to the volume average of the fluid velocity $\langle\mathbf{v}\rangle=\left\langle\mathbf{v}^{P}\right\rangle+\left\langle\mathbf{v}^{C}\right\rangle$ by the classical relation which is valid for single porosity media. When the material of the microporous matrix is incompressible

$$
\alpha=1, \quad \beta=0,
$$

it is easy to check from (49) and (50) that the corresponding effective Biot's parameters verify similar relations

$$
\alpha_{i j}^{e f f}=I_{i j}, \quad \beta^{e f f}=0 .
$$

Finally, let us recall that the macroscopic equivalent model was obtained under the conditions that stresses and pressures in both fluid and solid, as well as displacements in the fluid and the solid, are of similar order of magnitude.

\section{$5 \quad$ Numerical examples}

We investigate two different problems. Due to the symmetries of the period, the first one yields an isotropic macroscopic model. The second one investigates the case of flat penny-shaped cracks (an anisotropic orthotropic case).

\subsection{A porous medium with circulars vugs}

In order to study the influence of vugs on the poro-elastic behaviour of the porous material, let us consider a porous material which microstructure is presented in Figure 2. This microstructure was investigated in [18] with the assumption that the matrix is not porous, which is physically inconsistent with the assumption of the validity of Biot's model (no water flow is possible). We assume that the porous matrix material is isotropic and homogeneous. The poro-elastic parameters of the matrix are given in Table I.

The volumetric fraction of the vug is $\phi_{c}=0.126$. The determination of the macroscopic poroelasticity parameters $\alpha^{\text {eff }}$ and $\beta^{\text {eff }}$ consists in solving the local problems (28-29) and (30-31), followed by the application of the definitions (42) and (54). The computations were performed using the Comsol Multiphysics code and the Structural mechanics application mode (plane strain 


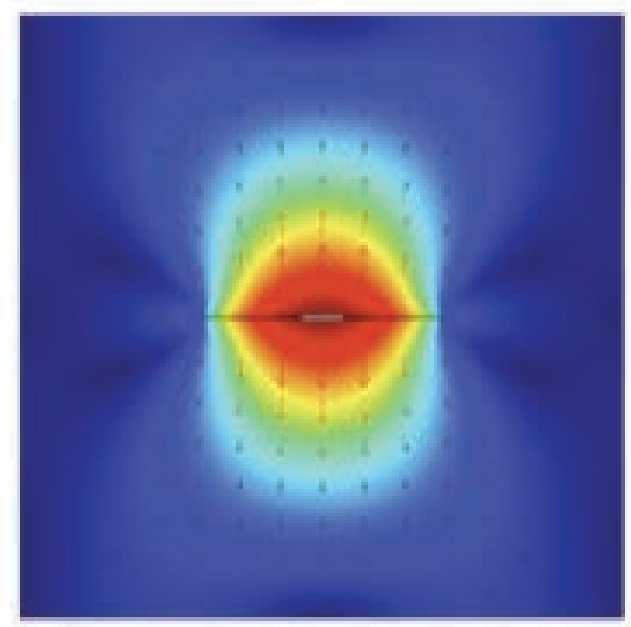

Figure 5: The solution $\boldsymbol{\xi}^{11}$ of the local problem (28-29) corresponding to a unit macroscopic strain in the $\mathrm{x}$-direction (horizontal).

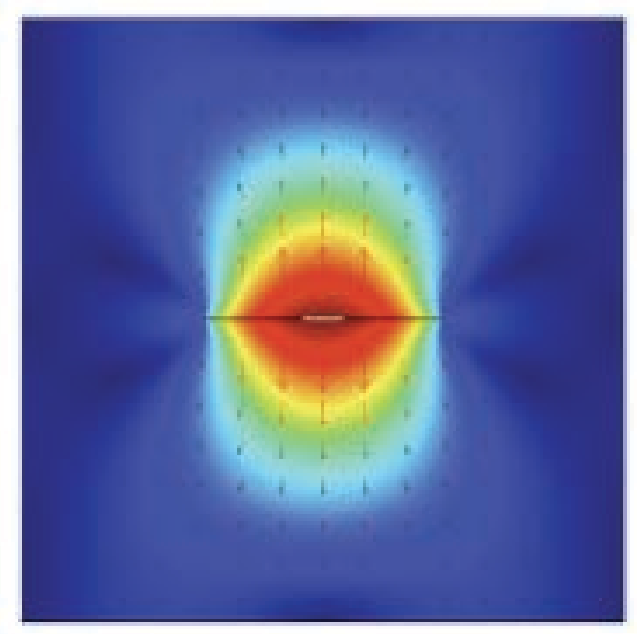

Figure 6: The solution $\boldsymbol{\xi}^{22}$ of the local problem (28-29) corresponding to a unit macroscopic strain in the y-direction (vertical). 


\section{Surface: Total displacement [m] \\ Height: Total displacement [m] \\ Deformation: Displacement}

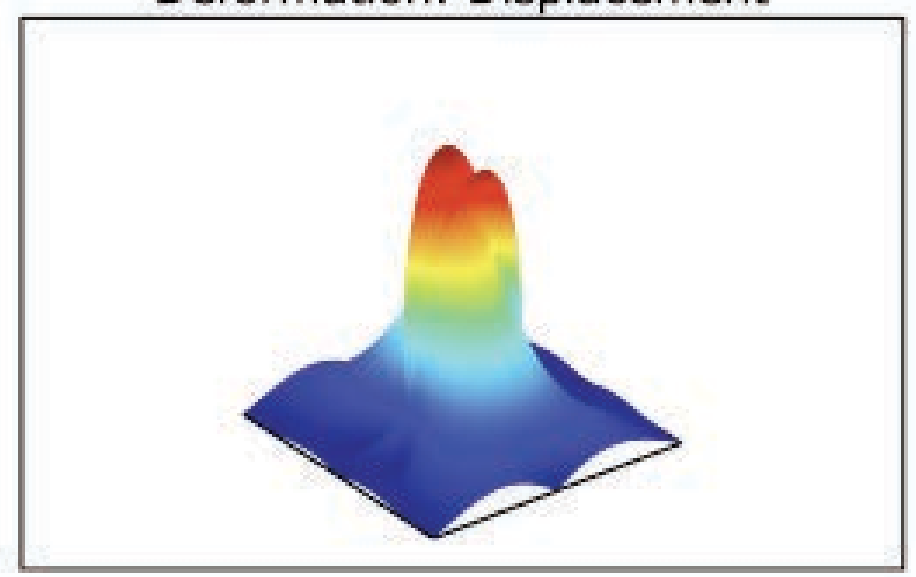

Figure 7: The solution $\boldsymbol{\eta}$ of the local problem (30-31) corresponding to $(1-\alpha) p^{(0)}=-1$ at the boundary $\Gamma$.

\begin{tabular}{|c|c|c|c|c|}
\hline Young modulus $E[\mathrm{GPa}]$ & Poisson ratio $\nu[]$ & Porosity $n[]$ & Biot parameter $\alpha[]$ & Biot parameter $\beta\left[G P a^{-1}\right]$ \\
\hline 1 & 0.3 & 0.126 & 0.334 & 0.218 \\
\hline
\end{tabular}

Table 1: Poro-elastic parameters of the porous matrix

conditions). Figure 3 represents the solution of the problem (28 -29). It shows the displacement field $\boldsymbol{\xi}^{11}$ corresponding to the case of a unit macroscopic strain in the x-direction (horizontal). Figure 4 presents the solution of the problem (30-31). We can see the displacement field $\boldsymbol{\eta}$ for which $(1-\alpha) p^{(0)}=-1$ at the boundary $\Gamma$. By using definitions (42) and (54) we obtained the following values of the poro-elastic Biot's parameters: $\alpha^{\text {eff }}=0.556$ and $\beta^{\text {eff }}=0.287$. It should be noticed that there are significant modifications of both parameters, if we compare these values with the values obtained in [18] for the case of the non-porous matrix, namely: $\alpha^{e f f}=0.334$ and $\beta^{e f f}=0.218$ (after correction of the sign in [18]). As expected both parameters are increased which means that the hydro-mechanical coupling becomes stronger. This example shows the importance of taking into account the porous nature of the matrix.

\subsection{A porous medium with flat cracks}

We consider the porous medium with the same parameters as in the example 5.1, but the crack is now of penny-shaped form and negligible volume. The semi-axes of the crack are 0.2 in the $\mathrm{x}$-direction and 0.002 in the $\mathrm{y}$ direction. The volumetric fraction of the crack is $\phi_{c}=0.0013$. The total porosity of the medium is almost the same as in the example 5.1, namely 0.127 . Two calculations were performed to solve the problem (28-29) by applying a macroscopic unit strain in the $\mathrm{x}$-direction and in the y-direction. Displacement $\boldsymbol{\xi}^{11}$ is presented in Fig. 5. As a first approximations the two lips of the crack can be considered as parallel to the $\mathrm{x}$ axis. Therefore the boundary condition (29) for $\boldsymbol{\xi}^{11}$ reads

$$
c_{i j k l} e_{y k l}\left(\boldsymbol{\xi}^{11}\right) n_{j} \approx-\lambda n_{2}=-\frac{E \nu}{(1+\nu)(1-2 \nu)} n_{2} \text { on } \Gamma
$$




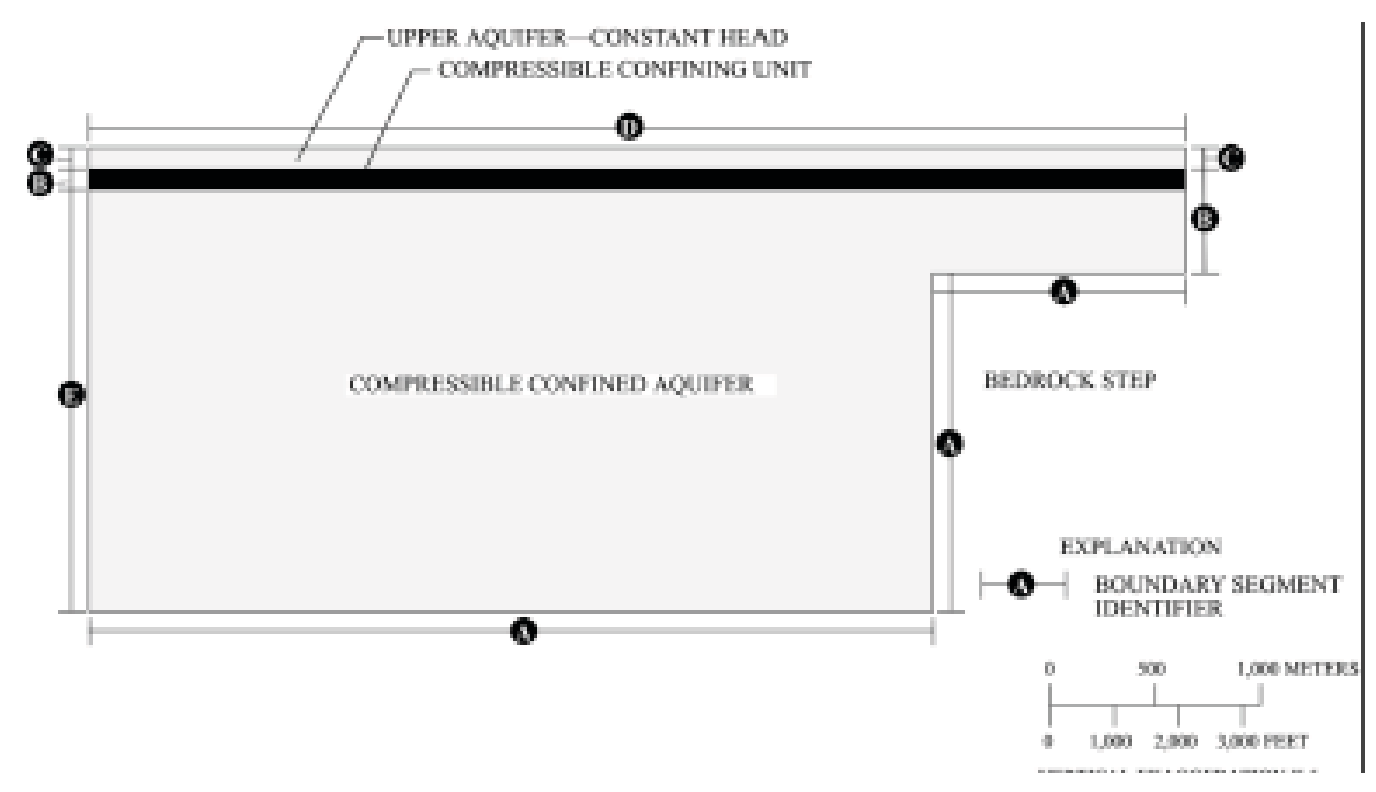

Figure 8: Model geometry with boundary conditions (after Leake and Hsieh, 1997)

whereas for $\boldsymbol{\xi}^{22}$ we have

$$
c_{i j k l} e_{y k l}\left(\boldsymbol{\xi}^{22}\right) n_{j} \approx-(\lambda+2 \mu) n_{2}=-\frac{E(1-\nu)}{(1+\nu)(1-2 \nu)} n_{2} \text { on } \Gamma,
$$

where $\lambda$ and $\mu$ are the Lamé constants. Therefore displacements $\boldsymbol{\xi}^{11}$ and $\boldsymbol{\xi}^{22}$ are approximately parallel, that can be observed in figures 5 and 6 and we have

$$
\frac{\left|\boldsymbol{\xi}^{22}\right|}{\left|\boldsymbol{\xi}^{11}\right|} \approx \frac{1-\nu}{\nu}
$$

The obtained values of Biot's parameters are $\alpha_{11}=0.404$ and $\alpha_{22}=0.497$. Note that both values are greater than Biot's parameter $\alpha=0.334$ of the porous matrix itself. The solution to the problem (30- 31) is given in Fig. 7. It can be observed that the lips of the crack overlap. However, remember that the displacement corrector $\mathbf{u}^{S(1)}$ is equal to $-\boldsymbol{\eta}$. Moreover, the actual displacement corrector is $\varepsilon \mathbf{u}^{S(1)}, \varepsilon \ll 1$. The calculated Biot's coefficient is $\beta^{e f f}=0.298$. Again, it is greater than Biot's coefficient $\beta=0.218$ of the porous medium itself.

\subsection{Solution to a particular boundary value problem}

In order to illustrate the importance of taking into account the corrections proposed in this paper a particular boundary value problem was solved. The example was inspired by the problem presented in [19] and [20]. The initial problem aims at demonstrating how the pumping of water can produce ground settlements and associated lateral fissures. Our additional objective is to show the quantitative difference in the results obtained for two different cases: i) case 1: without taking into account the presence of vugs in the matrix, and ii) case 2: with taking into account the presence of vugs in the matrix.

\subsubsection{Geometry and governing equations}

The same geometry and initial and boundary conditions as in [19] and [20], were considered. Three sedimentary layers overlay impermeable bedrock. A bedrock step is occurring as shown in Fig. 8. The sediment thickness is $420 \mathrm{~m}$ at $x=0$ and $120 \mathrm{~m}$ above the step (for $x>4000 \mathrm{~m}$ ). The top two layers are each $20 \mathrm{~m}$ thick. The macroscopic boundary value problem is the extended Biot model proposed in this paper, $(56,57)$. The boundary conditions are the same as in [19] and [20], 


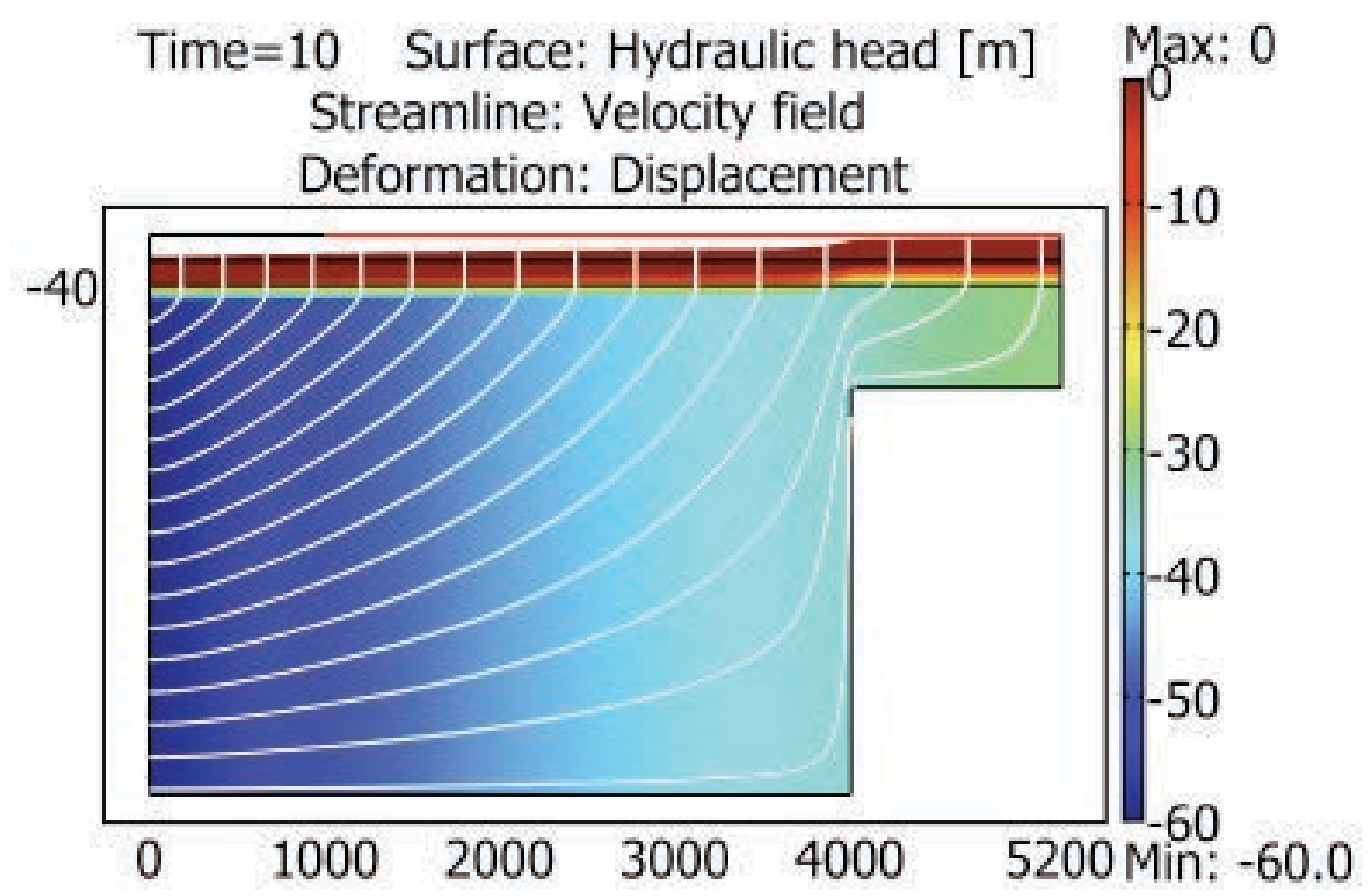

Figure 9: Case 2: the hydraulic head (colours), the fluid velocity field (arrows) and the fluid streamlines (lines) after 10 years of water pumping

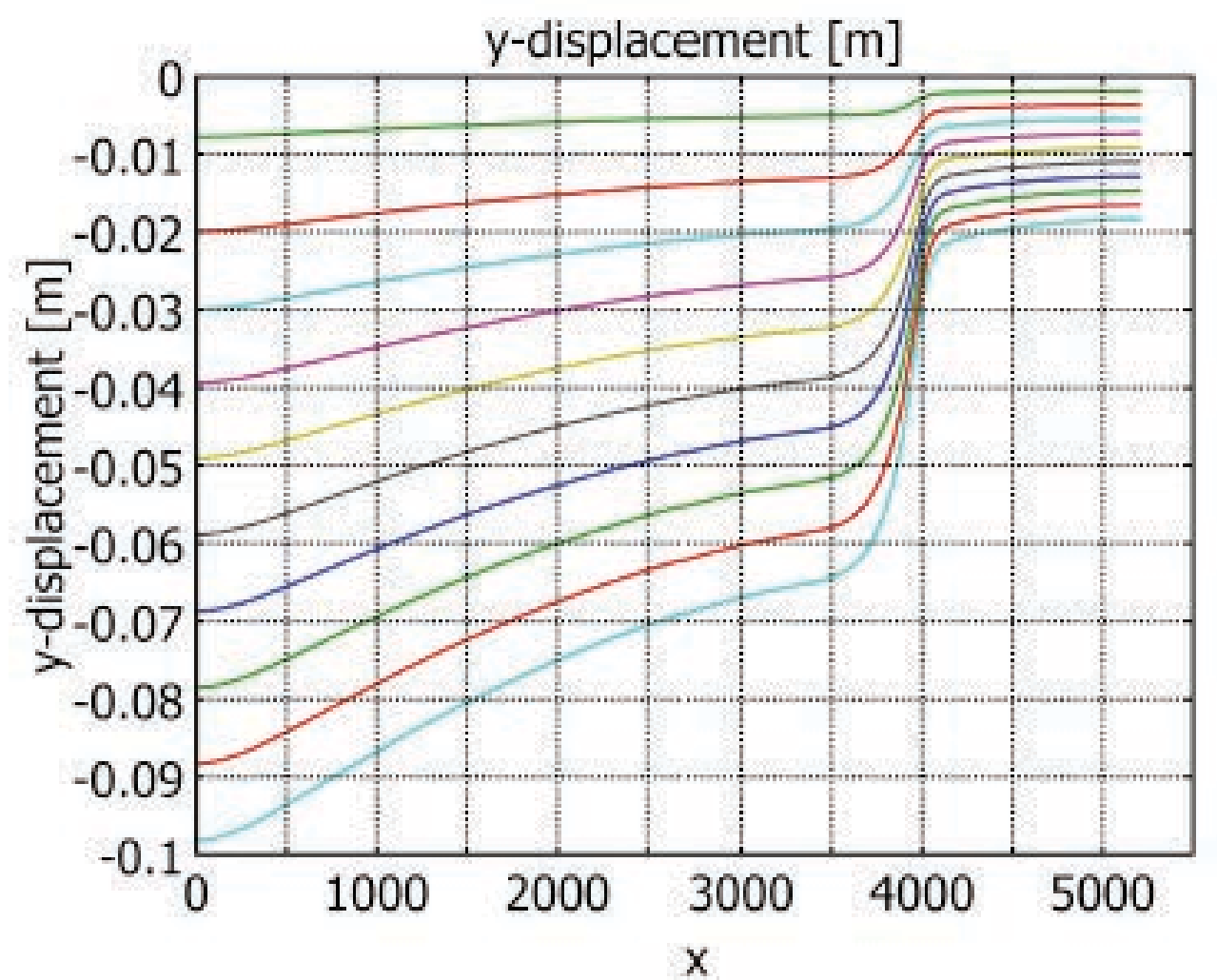

Figure 10: Case 1: the settlements of the surface ground $y=0$ for different times from 0 to 10 years 


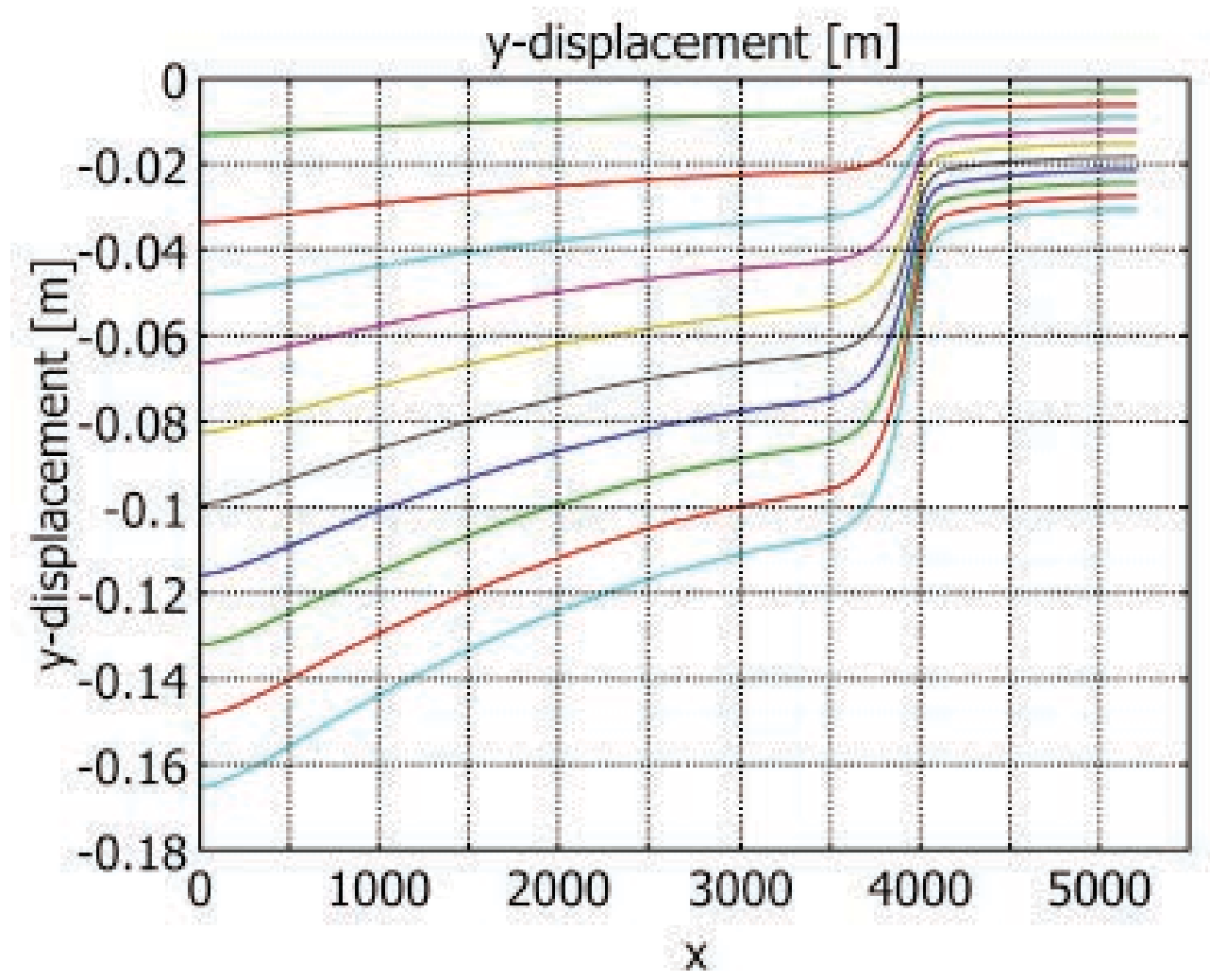

Figure 11: Case 2: the settlements of the surface ground $y=0$ for different times from 0 to 10 years

namely:

For the hydraulic problem:

n. $K \nabla H=0 \quad \partial \Omega \quad$ base $\quad$ A

n. $K \nabla H=0 \quad \partial \Omega$ other $\quad$ B

$H=H_{0} \quad \partial \Omega \quad$ upper edge $\quad \mathrm{C}$

$H=H_{0} \quad \partial \Omega \quad$ surface $\quad$ D

$H=H(t) \quad \partial \Omega \quad$ outlet $\quad \mathrm{E}$

where $H$ is the hydraulic head, $\mathbf{n}$ is the vector normal to the boundary. $H_{0}=0$ and $H(t)=6$ $(\mathrm{m} /$ year $) * \mathrm{t}$.

For the mechanical problem

$\begin{array}{llll}u=v=0 & \partial \Omega & \text { base } & \mathrm{A} \\ u=0 & \partial \Omega & \text { other } & \mathrm{B} \\ u=0 & \partial \Omega & \text { upper edge } & \mathrm{C} \\ \text { free } & \partial \Omega & \text { surface } & \mathrm{D} \\ u=0 & \partial \Omega & \text { outlet } & \mathrm{E}\end{array}$

where $u$ and $v$ are the components of the solid displacement vector.

\subsubsection{Model data.}

The water flow is initially at steady state but pumping from the lower aquifer reduces hydraulic head by $6 \mathrm{~m}$ per year at the profile $x=0$. The fluid supply in the upper reservoir is assumed limitless. The period of interest is 10 years. The materials are considered as homogeneous and isotropic within each layer. In Table 2 the model data are presented. The two sets of Biot parameters for the 


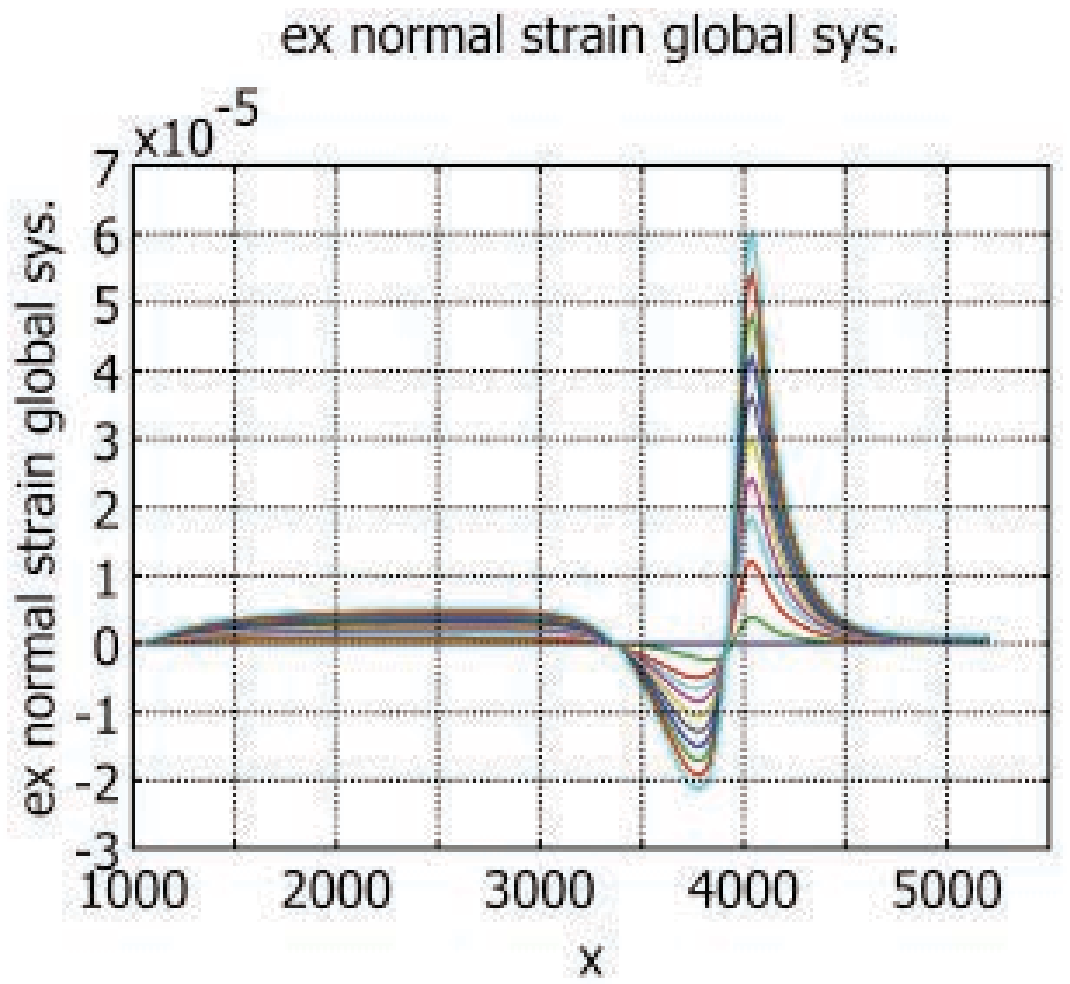

Figure 12: Case 1: The horizontal strain at the ground surface $y=0$ for different times from 0 to 10 years.

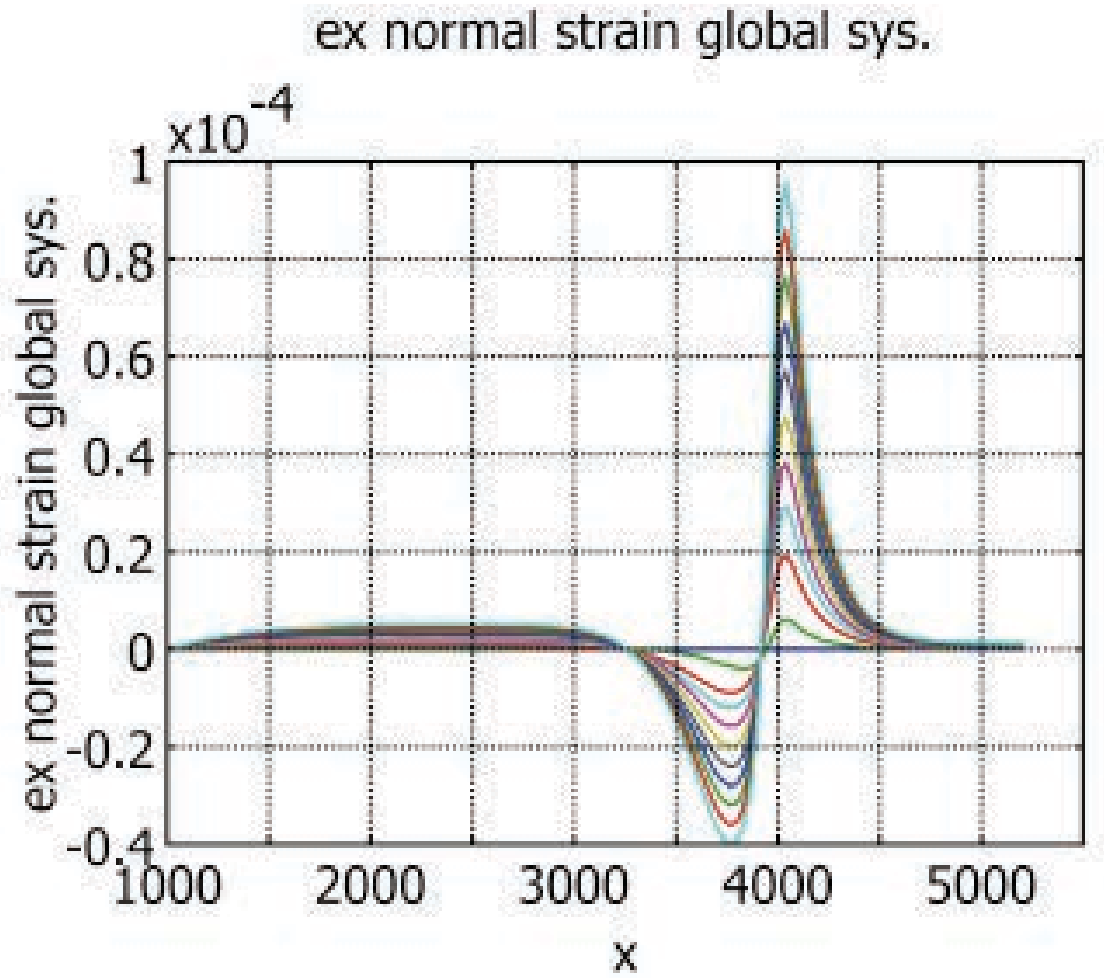

Figure 13: Case 2: The horizontal strain at the ground surface $y=0$ for different times from 0 to 10 years 


\begin{tabular}{|c|c|c|}
\hline Quantity & Upper and lower layer (aquifers) & Middle layer \\
\hline Hydraulic conductivity & $2.89 * 10^{-4} \mathrm{~m} / \mathrm{s}$ & $1.16 * 10^{-7} \mathrm{~m} / \mathrm{s}$ \\
\hline Fluid density & $1000 \mathrm{~kg} / \mathrm{m}^{3}$ & $1000 \mathrm{~kg} / \mathrm{m}^{3}$ \\
\hline Solid density & $2750 \mathrm{~kg} / \mathrm{m}^{3}$ & $2750 \mathrm{~kg} / \mathrm{m}^{3}$ \\
\hline Young modulus & $800 \mathrm{MPa}$ & $80 \mathrm{MPa}$ \\
\hline Poisson ratio & 0.25 & 0.25 \\
\hline Biot parameters: & & \\
\hline $\begin{array}{c}\text { Case 1: } \\
\text { without vugs (see 5.1) }\end{array}$ & $\begin{array}{c}\alpha=1 \\
\beta=1.02 * 10^{-10} \mathrm{~Pa}^{-1}\end{array}$ & $\begin{array}{c}\alpha=0.334 \\
\beta=2.73 * 10^{-9} \mathrm{~Pa}^{-1}\end{array}$ \\
\hline $\begin{array}{c}\text { Case } 2: \\
\text { with vugs }(\text { see } 5.1)\end{array}$ & $\begin{array}{c}\alpha=1 \\
\beta=1.02 * 10^{-10} \mathrm{~Pa}^{-1}\end{array}$ & $\begin{array}{c}\alpha=0.556 \\
\beta=3.59 * 10^{-9} \mathrm{~Pa}^{-1}\end{array}$ \\
\hline
\end{tabular}

Table 2: The model data

middle layer (case 1 and case 2) correspond to values of these parameters obtained from numerical computations presented in the subsection 5.1.

\subsubsection{Results.}

The problem was implemented into Comsol Multiphysics code [21], using Earth Science module and Structural Mechanics application mode (plane strain) of the standard set of options available in Comsol. The results concerning the hydraulic problem are very similar in both cases (Case 1 and Case 2). As an example, in Figure 9 the hydraulic head (colours), the fluid velocity field (arrows) and the fluid streamlines (lines) after 10 years for Case 2, are shown. It can be seen that the solution is as expected, with the maximum hydraulic head being negative and equal to $-60 \mathrm{~m}$ at $x=0$.

The difference in the solutions in two cases of Biot parameters for the middle layer can be seen, if we compare the settlements of the ground surface at $\mathrm{y}=0$. Case 1 is presented in Figure 10, while Case 2 is shown in Figure 11. The influence of the correction of the Biot parameters can also be seen when we compare the horizontal strains at the ground surface at $y=0$, Figures 12 and 13 . In both cases we observe negative strain (or compaction) on the left to the mountain step, and positive strains (or tension) on the mountain side. But the maximum values of these strains are different in each case. As pointed out in [20] these strains are related to the potential of fissuring or collapse. Thus, this example clearly shows the importance of the modelling proposed in this paper.

\section{Conclusion}

In this paper the macroscopic model of hydro-mechanical coupling for the case of a porous medium containing cracks or vugs, was developed. This model represents an extension of the classical Biot's model that was initially developed for a homogeneous isotropic elastic saturated porous medium. In the development we applied the asymptotic developments homogenization method. It 
was shown that the structure of the macroscopic equivalent model is similar to the Biot's model, but the poro-elastic parameters (the so called Biot's parameters), are modified. In the present investigation, Biot's parameters of the porous matrix itself, as well as the volumetric fraction of the cracks (vugs), are taken into account.

The numerical example showed quantitative differences in taking into account the presence of isolated cracks (or vugs) in a porous matrix. It should also be emphasized that the connectivity of the domains plays a primary role in the modelling of coupled phenomena. The micro-mechanical approaches offer the opportunity to capture this feature since the microstructure is given explicitly. The analysis presented in this paper can be easily extended to the case of local anisotropy of the porous medium.

\section{References}

[1] Levy T.1990. Filtration in a porous fissured rock: influence of the porous cnnexity Eur. J. Mech., B/Fluids, 9(4), 309-327.

[2] Lewandowska J., Szymkiewicz A., Burzynski K., Vauclin M. 2004. Modelling of unsaturated water flow in double-porosity soils by the homogenization approach Advances in Water Resources, 27, 283-296.

[3] Lewandowska J., Auriault J.-L. 2004. Modelling of unsaturated water flow in soils with highly permeable inclusions, C.R. Mecanique 332, 91-96.

[4] Lewandowska J., Szymkiewicz A., Auriault J.-L. 2005. Upscaling Richard's equation for soils containing highly conductive inclusions, Advances in Water Resources. 28, 1159-1170.

[5] Auriault J.-L., Boutin C. 1992. Deformable porous media with double porosity. Quasi-statics : I Coupling effects, T. I. P. M. 7, 63-82.

[6] Auriault J.-L., Boutin C. 1993. Deformable porous media with double porosity. Quasi-statics : II memory effects, T. I. P. M. 10, 153-169.

[7] Auriault J.-L., Boutin C. 1994. Deformable porous media with double porosity. Quasi-statics : iii acoustics, T. I. P. M. 14, 143-162.

[8] Barrenblatt G.I., Zheltov Y.P., Kochina I.N. 1960. Basic concepts in theory of seepage of homogeneous liquids in fissured rocks, P.M.M. 24, 852-864.

[9] Warren J.O., Root P.J. 1963. The behaviour of naturally fractured reservoirs, Soc. Petrol. Engng J. 1, . 245-255.

[10] Wilson R. K., Aifantis E. C. 1982. On the theory of consolidation with double porosity, Int. J. Engng. Sci., 20, 10091035.

[11] Sanchez-Palencia E. 1980. Non-homogeneous media and vibration theory, Springer-Verlag Berlin.

[12] Bensoussan A., Lions J.-L., Papanicolaou G. 1978. Asymptotic Analysis for periodic structures, North Holland, Amsterdam.

[13] Dormieux L., Kondo D. Ulm F-J. 2006. Microporomechanics, Wiley and Sons Ltd.

[14] Yuan S.C., Harrison J.P. 2006. A review of the state of the art in modelling progressive mechanical breakdown and associated fluid flow in intact heterogeneous rocks, Int. J. of Rock Mechanics and Mining Sciences, 43, 1001-1022. 
[15] Biot M. A., 1941. General theory of three dimensional consolidation J. Appl. Physics, 12, $155-164$.

[16] Biot M.A. 1955. Theory of elasticity and consolidation for a porous anisotropic solid, J. Appl Physics 26, 182-185.

[17] Auriault J.-L. 2005. Transport in porous media : upscaling by multiscale asymptotic expansions, in CISM Lectures 480 Applied micromechanics of porous materials, Udine 19-23 July 2004, L. Dormieux and F.-J. Ulm EDS, pp 3-56, Springer 2005.

[18] Lydzba D., Shao J.F. 2000. Study of poroelasticity material coefficients as response of microstructure, Mech. Cohes.-Frict. Mater. 5, . 149-171.

[19] Biot Poroelasticity in the Library of the Comsol Multiphysics . Code [21]

[20] Leake S.A., Hsiek P.A. 1997. Simulation of deformation of sediments from decline of groundwater levels in an aquifer underlain by a bedrock step, US Geological Survey, Open file report, $97-47$.

[21] Comsol Multiphysics . http://www.comsol.com 